\title{
EchoGéo
}

15 | 2011

La Belgique, État prospère en décomposition au cœur de l'Europe du nord-ouest

\section{Paysages de la Belgique et représentation idéologique}

\section{Christian Vandermotten et Claire Neuray}

\section{OpenEdition}

Journals

Édition électronique

URL : https://journals.openedition.org/echogeo/12323

DOI : $10.4000 /$ echogeo.12323

ISSN : 1963-1197

Éditeur

Pôle de recherche pour l'organisation et la diffusion de l'information géographique (CNRS UMR 8586)

Référence électronique

Christian Vandermotten et Claire Neuray, «Paysages de la Belgique et représentation idéologique », EchoGéo [En ligne], 15 | 2011, mis en ligne le 11 avril 2011, consulté le 03 août 2021. URL : http:// journals.openedition.org/echogeo/12323; DOI : https://doi.org/10.4000/echogeo.12323

Ce document a été généré automatiquement le 3 août 2021.

EchoGéo est mis à disposition selon les termes de la licence Creative Commons Attribution - Pas d'Utilisation Commerciale - Pas de Modification 4.0 International (CC BY-NC-ND) 


\title{
Paysages de la Belgique et représentation idéologique
}

\author{
Christian Vandermotten et Claire Neuray
}

\section{NOTE DE L'ÉDITEUR}

La localisation des photographies présentées dans ce texte est indiquée sur la carte publiée dans l'introduction de la rubrique Sur le Champ.

1 La description des paysages, leur organisation en ensembles typologiques et leur représentation photographique, fondements de la géographie régionale traditionnelle, relèvent-elles de l'objectivité scientifique dont se prévalaient les Vidaliens ou de la transmission de constructions subjectives et de représentations idéelles?

2 Pourquoi ne construit-on pas une géographie régionale sur la base de l'analyse (fondée sur quels critères?) d'un ensemble de photographies prises systématiquement, par exemple aux coins d'une grille de $5 \mathrm{~km}$ de côté, quitte à photographier le parking d'un supermarché, un commerce d'automobiles le long d'une grand-route ou un pavillon périurbain? Cela n'est pas fait et pourtant ces objets peuvent aussi donner lieu à une analyse géographique. Et encore, choisirait-on des photos prises au grand angle ou non, privilégiant une direction de prise de vue ou pas, etc. ?

3 Le présent dossier doit donc être lu - regardé - non comme l'expression d'une nostalgie vidalienne, mais comme un clin d'oeil critique, comme un exercice qui ne peut être que subjectif, qui s'interroge en filigrane sur les représentations spatiales privilégiées: le choix des photos sensées illustrer la diversité d'un territoire, ici la Belgique, et sa régionalisation géographique reste déterminé largement par la subjectivité d'une vision qui privilégie les permanences, les paysages supposés naturels, en fait ruraux, mais plutôt la ruralité d'une prairie et d'une ancienne haie que celle d'un élevage hors sol ou d'un chemin de remembrement. En fait, l'identification des régions agricoles et des archétypes paysagers qui y sont associés apparaît dans la seconde moitié du XIX ${ }^{\mathrm{e}}$ siècle, en réponse aux progrès de la géologie et des sciences agronomiques et justement 
à partir du moment où l'expansion du marché national des produits agricoles et des transports rend importante la connaissance (et la promotion) des ressources spécifiques de chaque portion de l'espace national, mais contradictoirement commence à développer les conditions de sa banalisation. C'est aussi l'époque où la bourgeoisie romantique découvre la nature, supposée saine et terreau dans lequel s'inscrit le sentiment national, expression de l'unité dans la diversité.

Nous parcourrons ci-après, pour inciter entre les lignes à les déconstruire, quelques paysages belges, tout aussi subjectivement choisis que les photos qui les illustrent; le biais est clair, il s'agira avant tout des paysages valorisés, de ceux où on se promène, pour "prendre l'air", dans la "nature», de ceux que privilégient - et que marchandisent - les guides touristiques. Même si quelques vues narquoises montreront d'autres facettes, ce ne sera pas la Belgique des interminables lotissements de villas quatre façades, précédées de leur pelouse ou de leur aire de stationnement en graviers, de leur petit nain de jardin et de leur araucaria ridicule, que l'on peut observer le long des routes entre deux stations d'essence et deux parkings d'implantations commerciales. Et pour les paysages présentés, le photographe a sans doute fait bien souvent le pas de côté qui lui permettait d'éviter sur son cliché le poteau d'éclairage, la maison moderne ou la voiture en stationnement, y compris la sienne.

Enfin, conseillons à ceux qui voudraient parcourir plus en détail, et dans une perspective historique longue, les paysages belges, ruraux, mais tout autant urbains ou industriels, l'ouvrage de M. Antrop, Ph. De Maeyer, C. Vandermotten, M. Beyaert \& al. (2006), La Belgique en cartes. L'évolution du paysage à travers trois siècles de cartographie (mais aussi de gravures et de photographies), Bruxelles, Institut géographique national et Tielt, Lannoo.

\section{La côte belge et son arrière-pays}

Espace privilégié du tourisme belge, la côte étire sur $66 \mathrm{~km}$, de la frontière française à celle des Pays-Bas, une plage de sable, longée par la plus longue ligne de tramway d'Europe. Espace de rêve, de contemplation de l'horizon et de l'infini, de nature saine, auquel beaucoup préfèrent les gaufres, les cuistax - petites autos à pédales, les magasins de la digue et les dancings. A l'arrière des plages, les dunes offrent des paysages que l'on peut croire sauvages si on s'abrite bien dans le creux d'une panne. Derrière le cordon dunaire, les polders maritimes, construits et aménagés depuis le Moyen âge, parcourus par des canaux de drainage ou de navigation, parfois bordés d'alignements de peupliers, valorisent pour les cyclistes qui s'aventurent à partir des stations balnéaires une "authenticité » fondée sur des densités de population faibles par rapport à la moyenne flamande, sur quelques grosses fermes d'abbaye ou sur les souvenirs de la navigation médiévale sur le Zwin, dont l'estuaire s'avançait jusque Damme, avant-port de Bruges. Charles de Coster (1827-1879) y a fait naître Tijl Uilenspiegel $^{1}$, luttant contre «l'occupation» espagnole, héros d'une version romantique libérale, "belge», anti-catholique, truffée de symbolisme maçonnique, d'une légende que l'on trouve ailleurs dans le monde germanique. 
Illustration 1 - Coucher de soleil sur la plage à De Haan (Le Coq). Rêve et contemplation

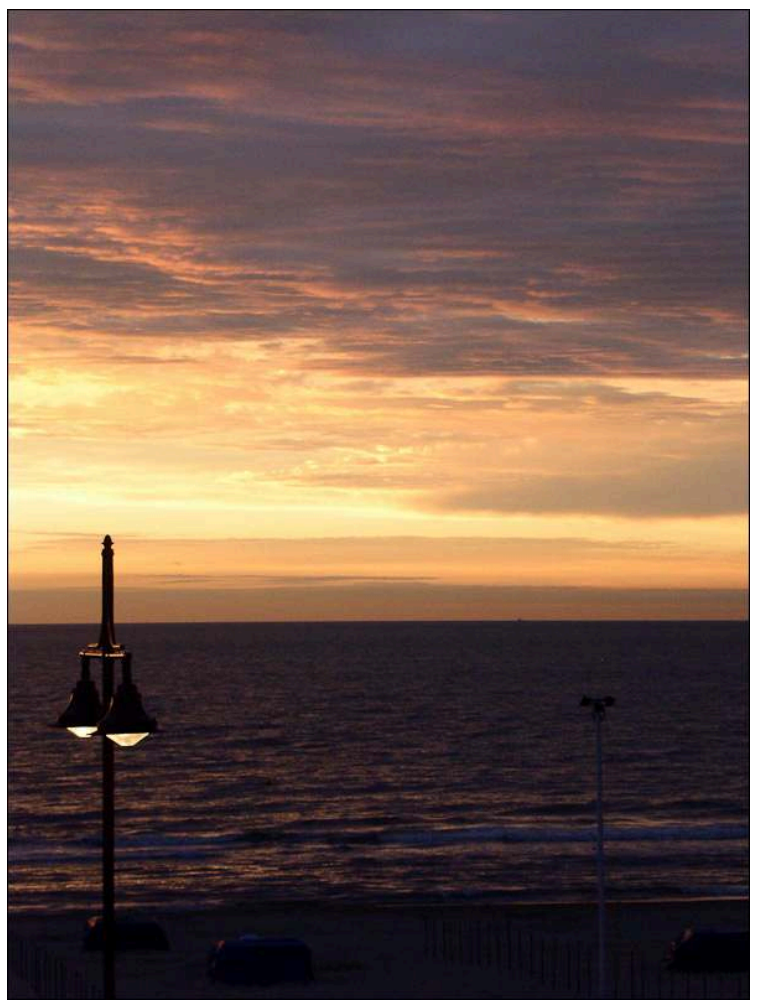

Auteur : C. Vandermotten, 2005

Illustration 2 - Blankenberge (la montagne = la dune blanche), au mois d'août

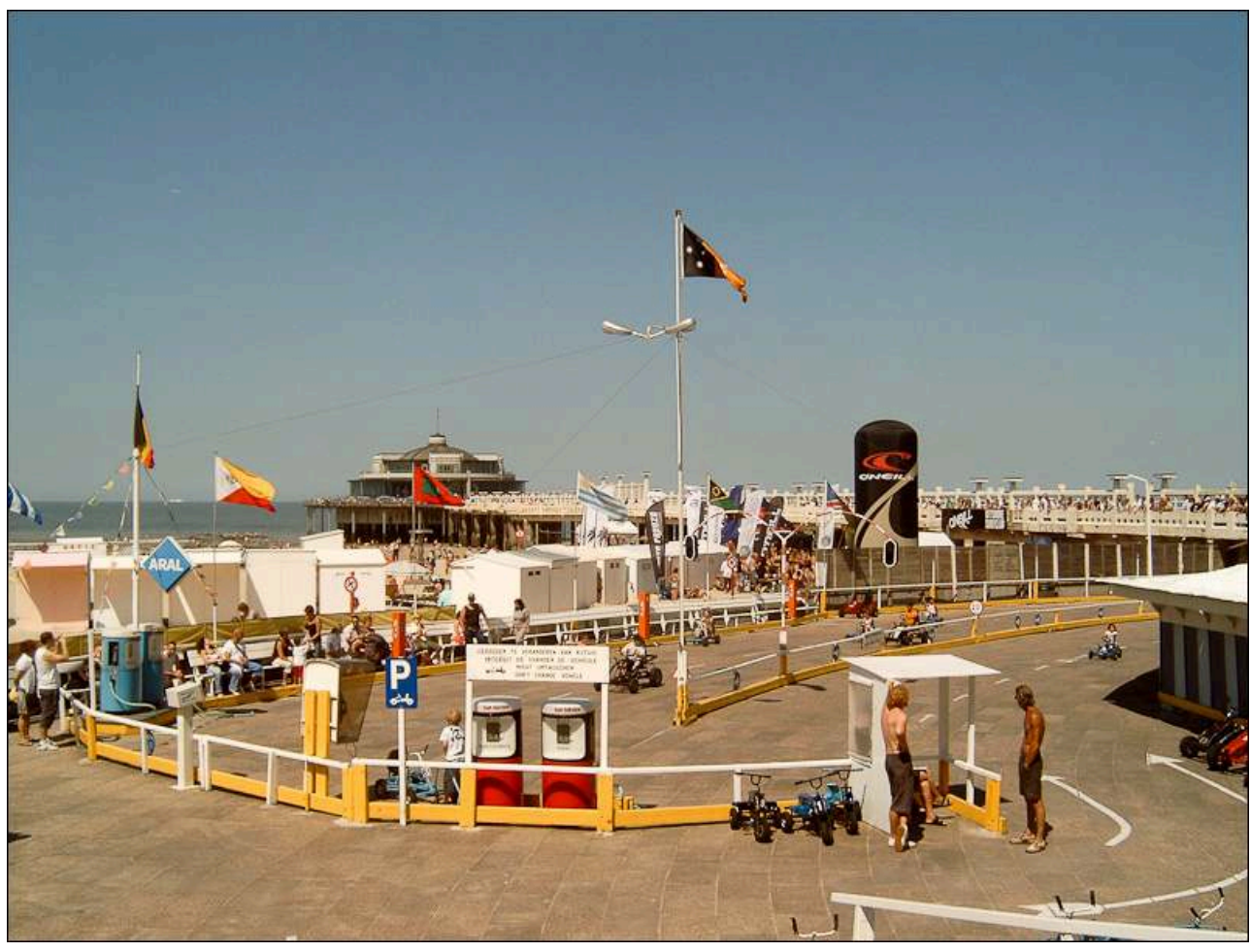

L'estacade du Pier s'avance dans la mer.

Auteur: Michielverbeek, 2007 
Illustration 3 - La Doornpanne (la panne - dépression intradunaire - des épineux) à Oostduinkerke. Vieilles dunes fixées

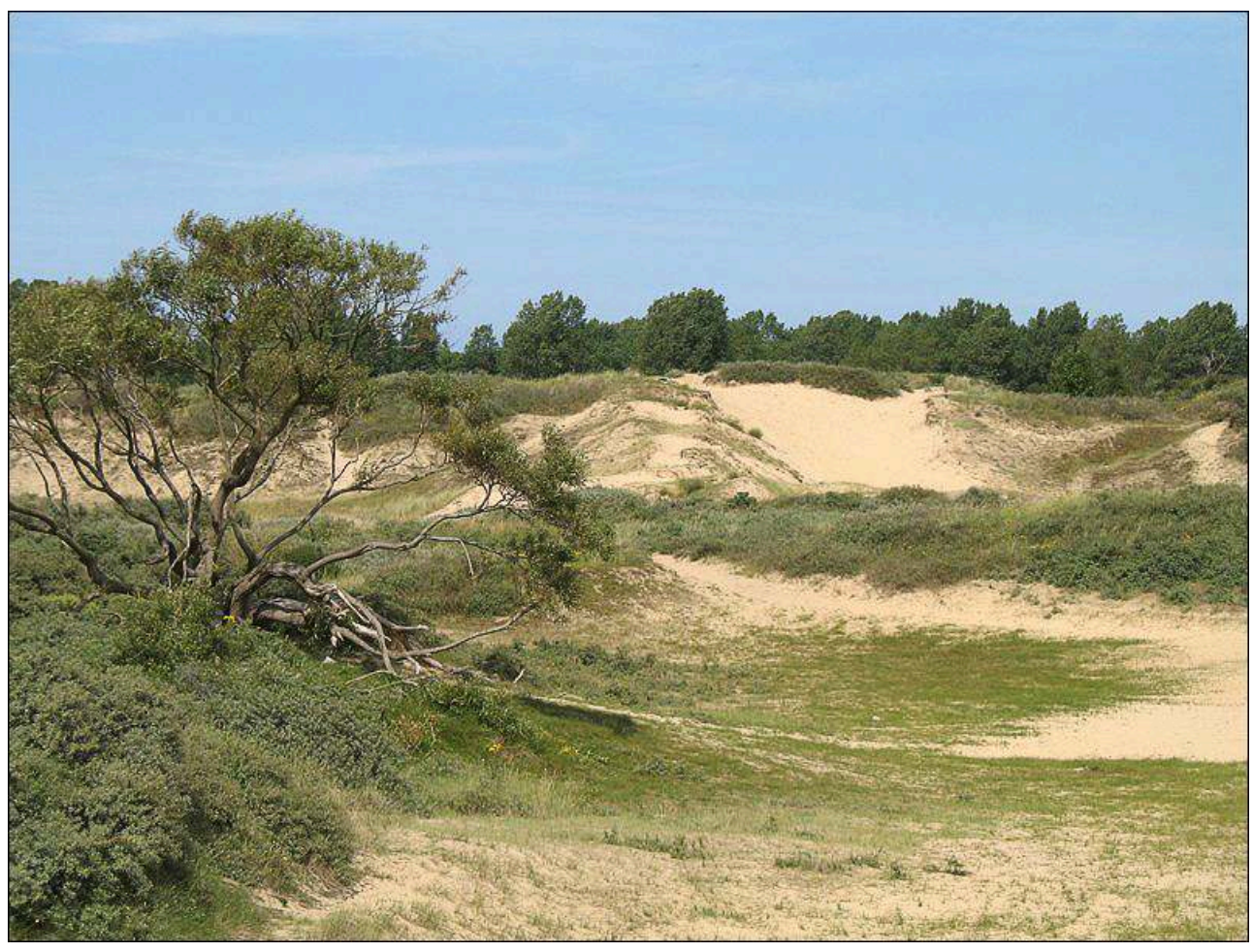

Auteur: M. Ryckaert, 2007. 
Illustration 4 - Le canal de Sluis à Bruges, au nord de Damme, près de la frontière néerlandaise

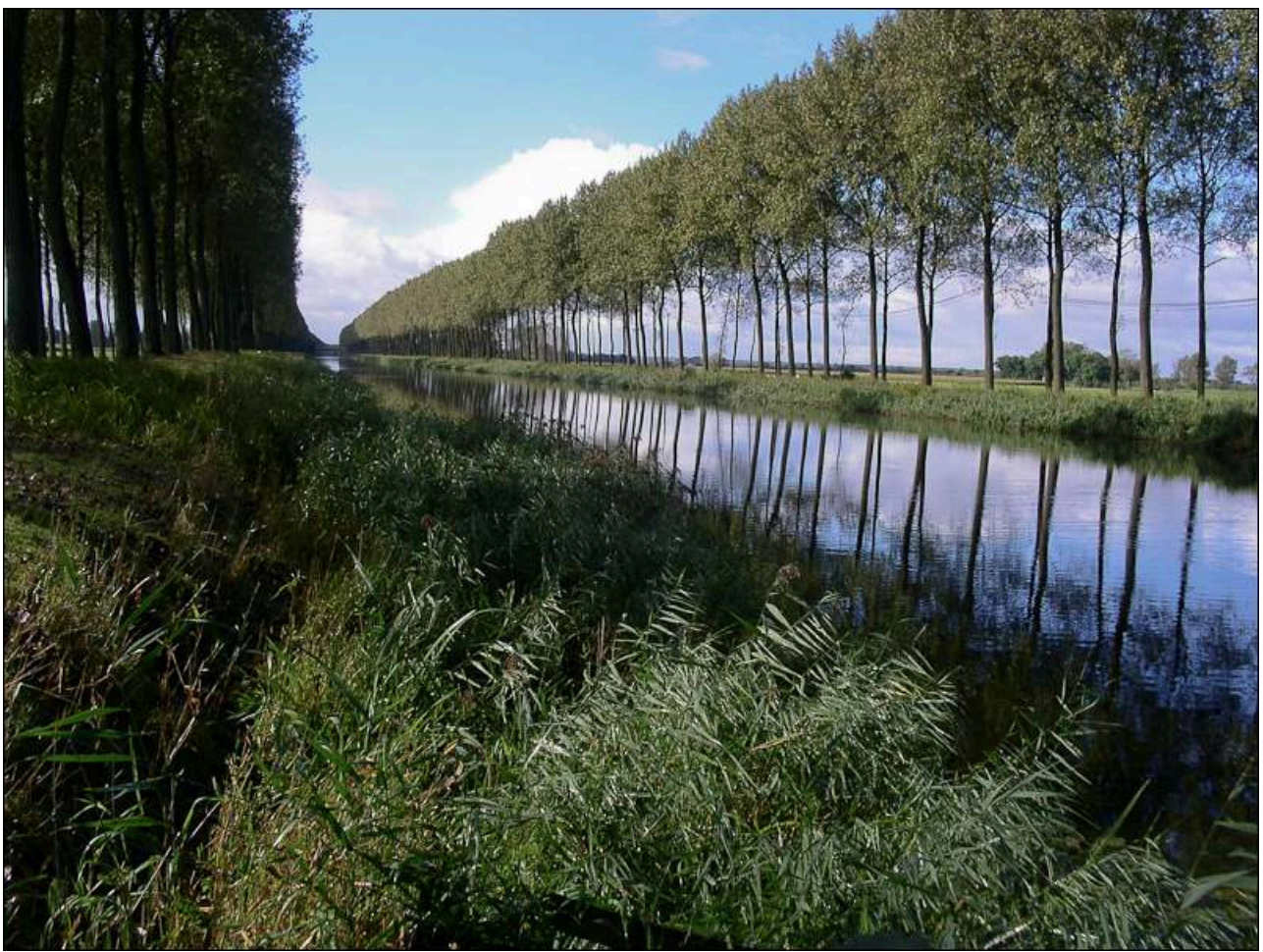

C'est peut-être là qu' « avec un ciel si bas un canal s'est perdu » (Jacques Brel, 1962). Bordé de peupliers d'une belle dimension verticale qui se reflètent dans le miroir de l'eau calme, le canal incite à la promenade le long de ses berges. En coulisse, derrière le rideau des troncs espacés, les étendues planes des polders se devinent. Si l'horizontalité caractérise la grande majorité des paysages en Belgique, c'est dans cette plaine qu'elle se remarque surtout. Ici, les terres argileuses, peu perméables, sont plus basses que le niveau de la mer et les cours d'eau ne créent pas de relief; au contraire, l'eau doit en être évacuée par tout un réseau de fossés de drainages, circulant presqu'à fleur du sol, à l'image de ce canal.

Auteur : C. Vandermotten, 2005 
Illustration 5 - Damme. L'hôtel de ville (1464)

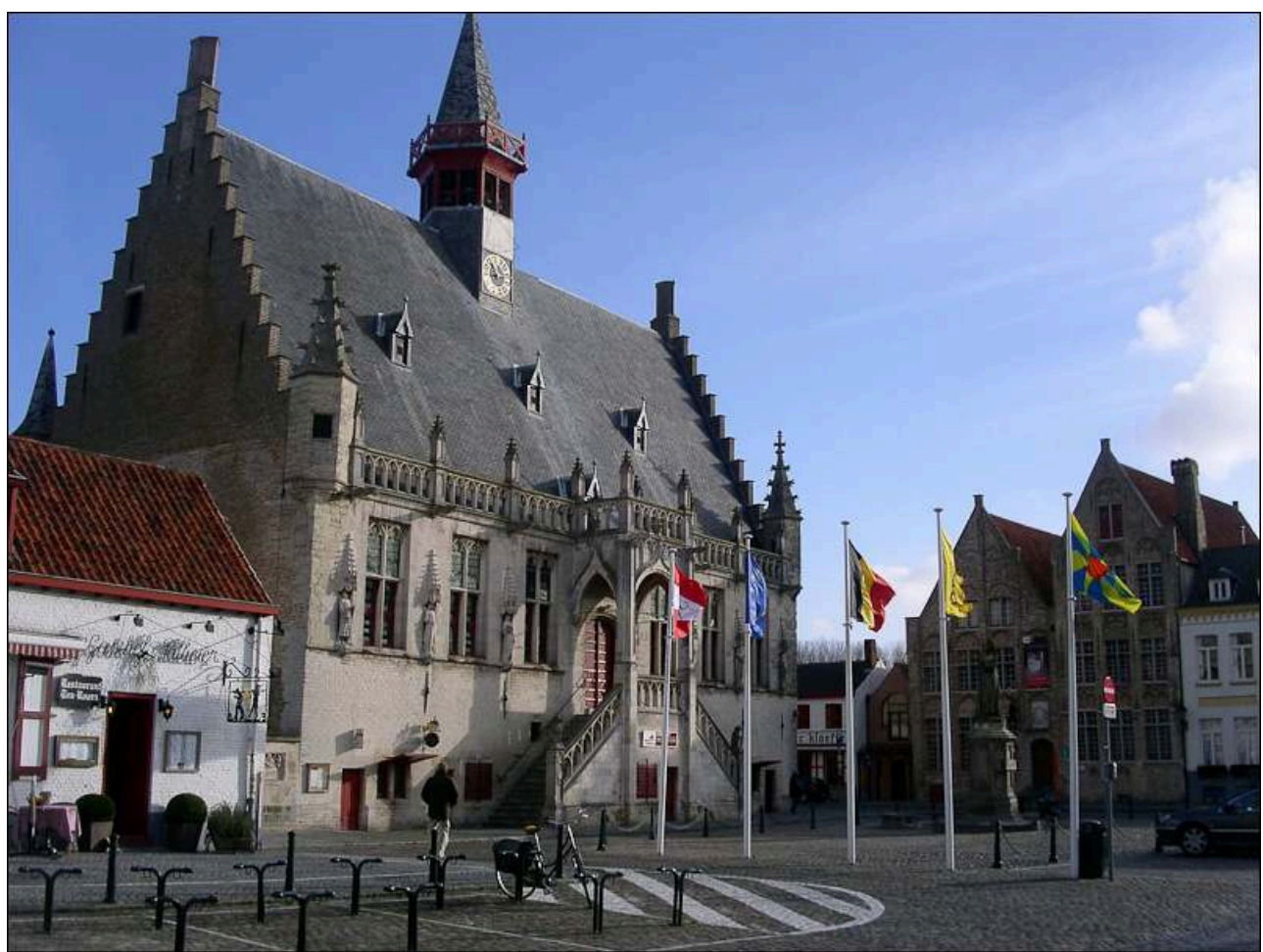

Les drapeaux témoignent de la complexité des références d'appartenance : Damme, l'Europe, la Belgique, la Région flamande, la province de Flandre occidentale.

Auteur : C. Vandermotten, 2007

\section{La Flandre intérieure et la Région des collines}

7 Le photographe contemporain ne pourrait plus illustrer l'image de la Flandre agricole intensive et laborieuse, celle du paysan têtu travaillant à la bêche, qu'en cadrant très soigneusement son cliché et en louant les services d'un figurant. C'est un peu plus aisé peut-être - du moins pour ce qui concerne le cadrage - dans la Région des collines, transition paysagère entre les basses terres flamandes et les bas-plateaux de la Moyenne Belgique wallonne, marquée par une série de collines, alignées d'ouest en est, où les densités de population sont un peu plus faibles. On y est un peu à l'écart des régions d'urbanisation et d'industrialisation diffuses généralisées que l'on trouve entre Courtrai et Gand ou dans l'espace métropolitain central belge, le "diamant flamand », un losange dont les sommets sont Gand, Anvers, Louvain et Bruxelles. 
Illustration 6 - La Flandre intérieure photographiée par le biologiste Massart au début du XX ${ }^{\mathrm{e}}$ siècle, ici à Overmere

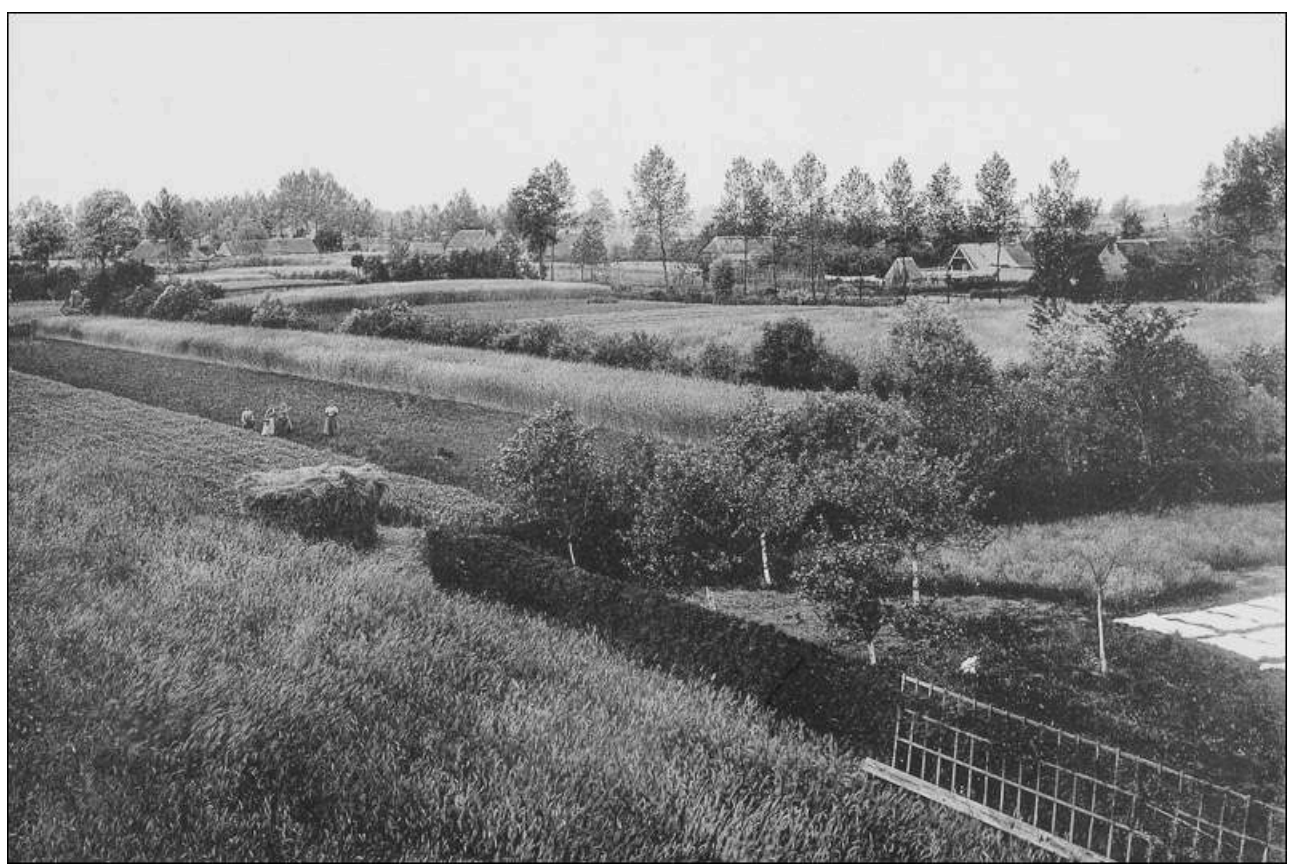

Les champs bombés, résultant des pratiques culturales à la bêche, étaient caractéristiques du Pays de Waes et y sont encore parfois reconnaissables.

Auteurs : Ch. Bommer et J. Massart, 1912.

\section{Illustration 7 - Près de Flobecq, village wallon en bordure de la frontière linguistique}

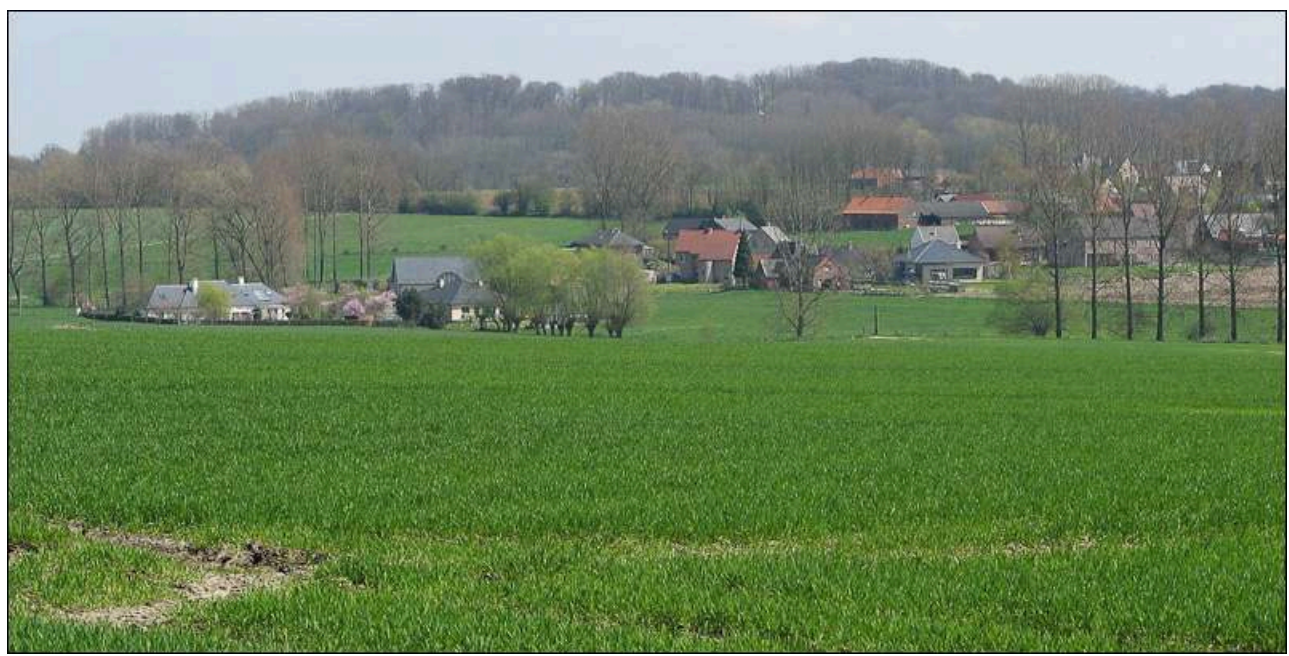

Les reliefs, aux sommets et versants souvent boisés, ont été protégés de l'érosion par suite de la transformation des sables sous-jacents en grès, plus résistants. Le travail du textile à domicile complétait ici, comme ailleurs en Flandre intérieure, les revenus de l'agriculture, ce qui a permis l'établissement d'une population importante dans de nombreux hameaux et fermes isolées.

Aujourd'hui, cet habitat, aux murs de briques et toits de tuiles rouges, est utilisé comme résidences secondaires ou principales. Il est complété par de nouvelles constructions pour des occupants qui bien souvent travaillent dans la capitale bruxelloise. Les éléments végétaux, tels les alignements d'arbres, peupliers et saules, y participent à l'image de campagne agricole, relativement protégée, du Parc naturel du Pays des collines, à l'authenticité duquel contribue la reconstruction de légendes sur des sorcières locales.

Auteur: C. Neuray, 2006. 


\section{Les landes de la Campine pauvre et infertile}

Les sols sablonneux du bas-plateau de la Campine n'étaient pas propices à la mise en culture. De vastes étendues planes, localement animées par des dunes et couvertes de bruyères, ont longtemps formé l'essentiel du paysage. Dans la seconde moitié du XIX ${ }^{e}$ siècle, la mise en valeur de ces terres de parcours de troupeaux, suite à l'aliénation des communaux, s'est concrétisée par la plantation d'arbres, surtout des conifères. Aux étangs et marécages qui se sont développés là où les indurations ferrugineuses imperméabilisent les sols, se sont ajoutés les vastes plans d'eau qui ont envahi d'anciennes sablières, suite au développement de l'industrie verrière à partir du début $\mathrm{du} \mathrm{XX}^{\mathrm{e}}$ siècle. S'il n'y avaient des réserves naturelles, on aurait bien de la peine à photographier encore une représentation traditionnelle des paysages de la Campine: d'abord attaquées par le développement des charbonnages à partir de la Première Guerre mondiale, les landes, et dans une moindre mesure les pinèdes, ont cédé la place à une industrialisation fordiste consommatrice d'espace, accompagnée d'une urbanisation diffuse, à partir des années soixante. Une industrialisation qui recherchait alors une main-d'œuvre abondante, peu syndicalisée, encadrée par l'Eglise.

Illustration 8 - La Campine des réserves naturelles.

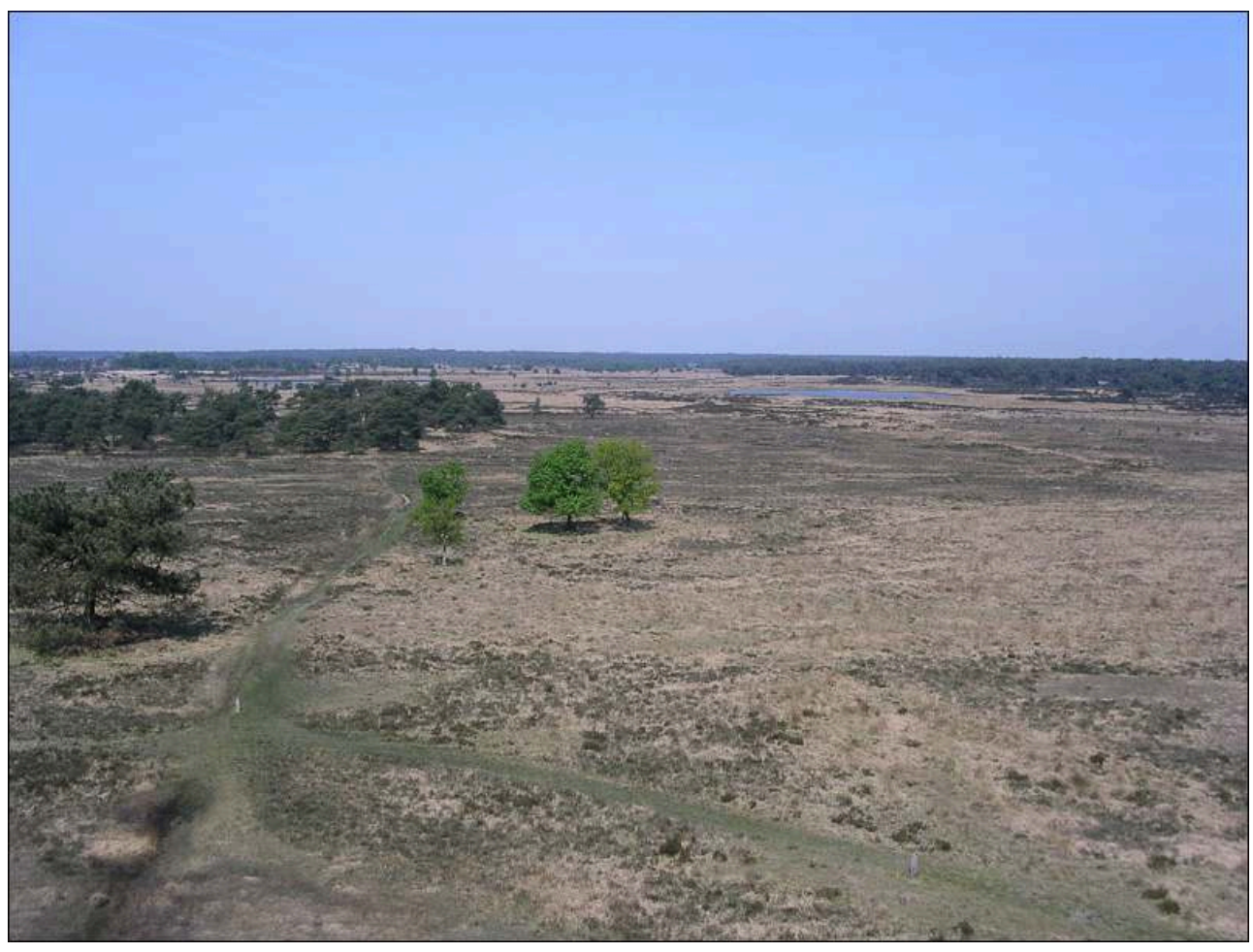

La Kalmthoutse heide (heide = lande ou bruyère), au nord d'Anvers, depuis le sommet d'une tour de surveillance contre l'incendie

Auteur: C. Vandermotten, 2007. 
Illustration 9 - Beringen

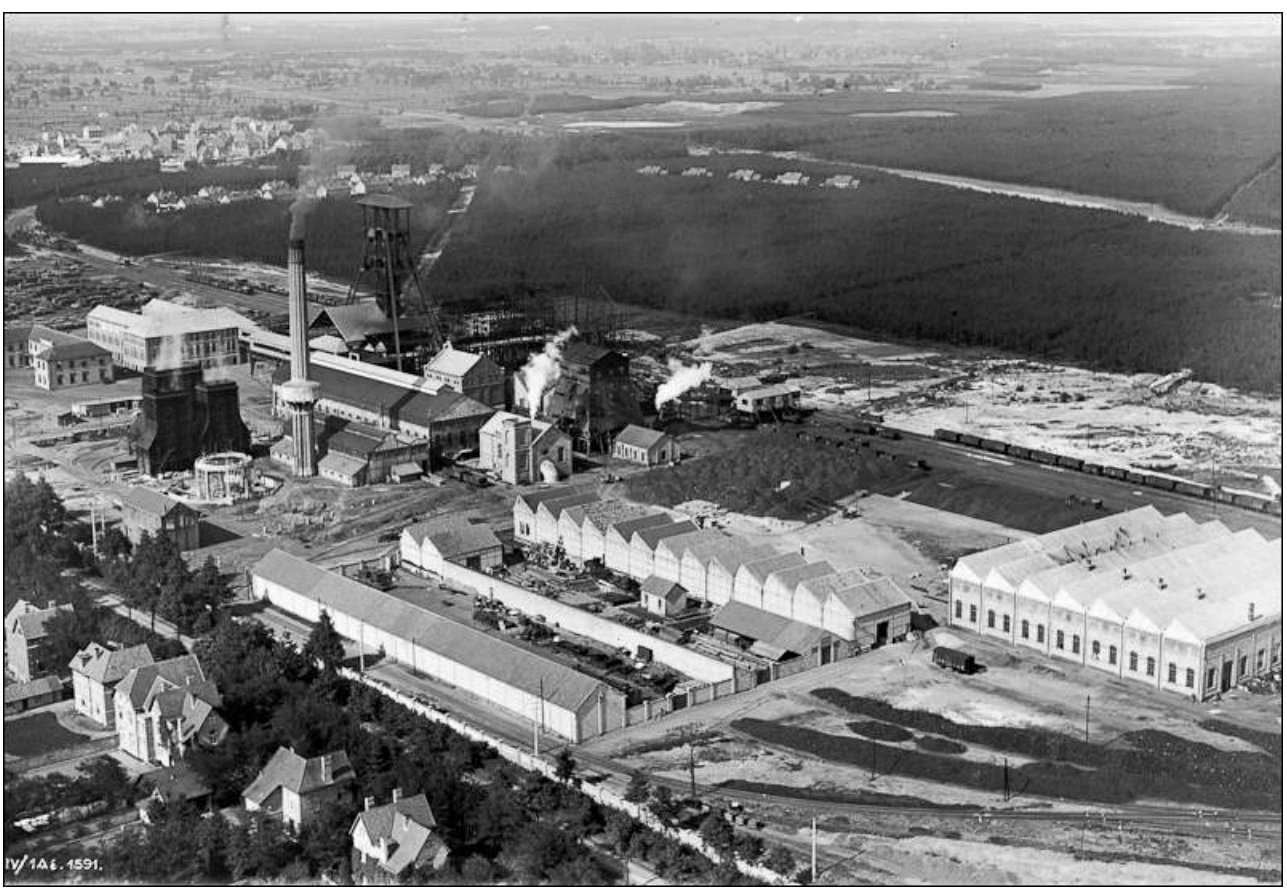

A partir des années 1920, le développement des charbonnages commence à entamer les pinèdes et la lande. L'industrie extractive s'est développée sur le plateau campinois beaucoup plus tardivement qu'en Wallonie, le premier puits n'étant mis en exploitation qu'en 1917. La prospection de ce gisement enfoui à grande profondeur n'avait débuté qu'au début du XX`e siècle. Dans cette région pauvre en ressources, peu peuplée et relativement isolée, des cités-jardins pour les mineurs et des villas pour le personnel de cadre ont été construites à proximité des installations du charbonnage et du puits d'extraction, bien identifiable grâce à la tour du châssis à molettes et à la grande cheminée.

Source : Musée de l'Aviation, vers 1930 
Illustration 10 - Lommel

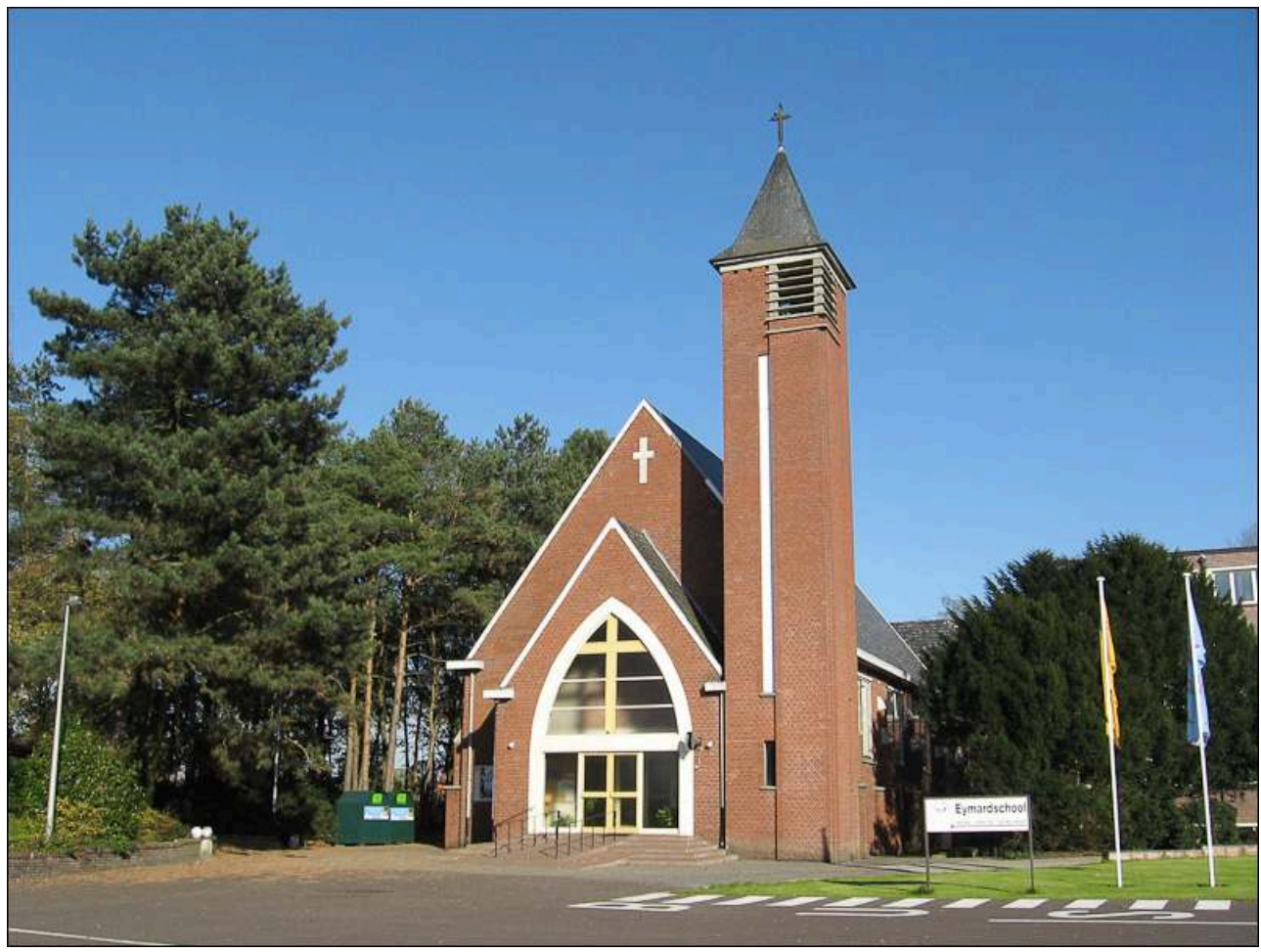

L'église du Saint-Sacrement (1939). La modernité et l'urbanisation diffuse pionnière qui ont triomphé de la lande transformée en pinède étaient socialement bien encadrées.

Source : Doyenné de Lommel (s.d.). 
Illustration 11 - Implantation industrielle à Genk

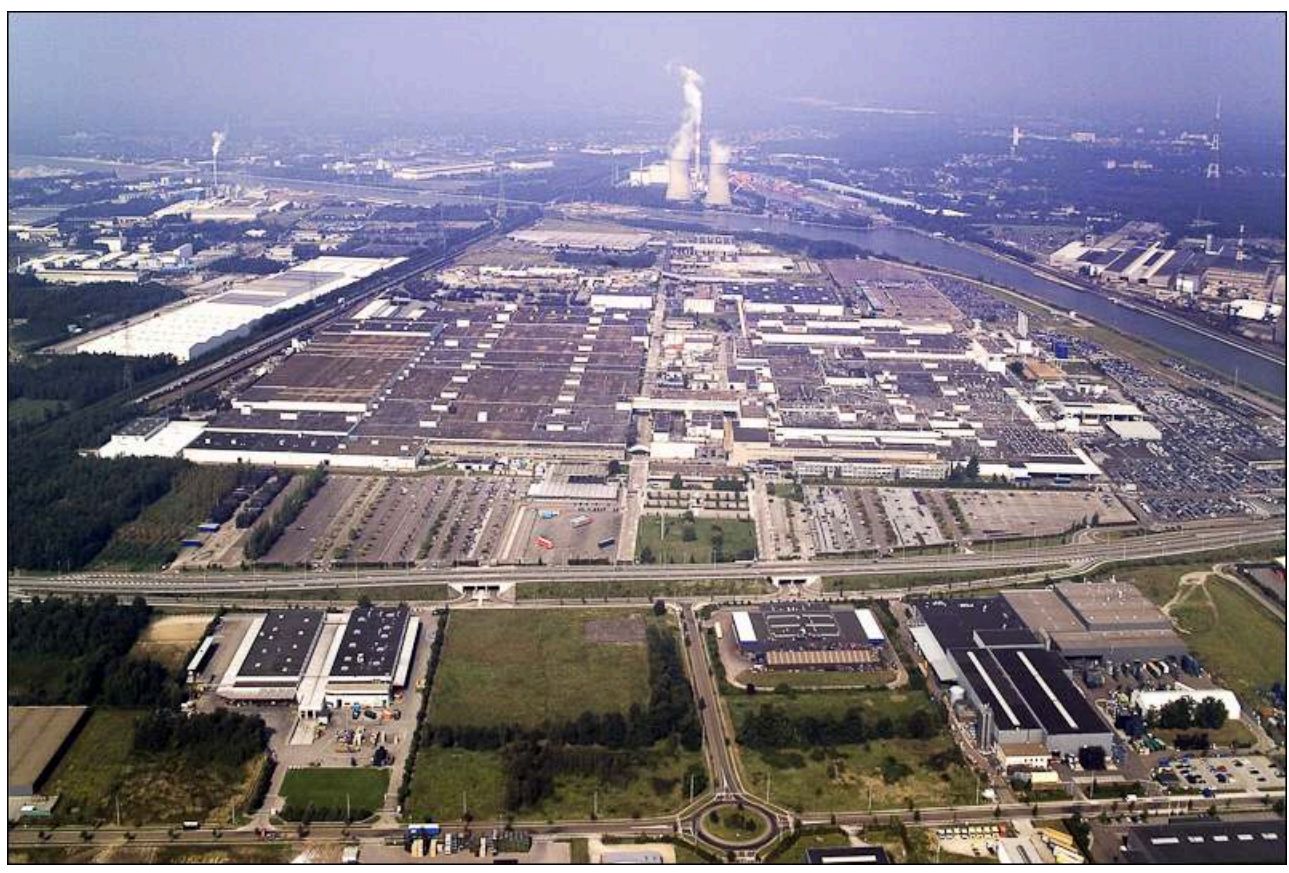

Au début des années 1960, Ford profite des nouvelles lois d'expansion économique attractives pour les capitaux étrangers pour s'implanter à Genk, sur de vastes étendues planes, couvertes de conifères et de prairies, en bordure du canal Albert. Une main-d'œuvre importante et sans trop de qualification y est utilisable pour le travail sur les chaînes de montage installées dans de longs bâtiments de faible hauteur. Au départ de cet énorme complexe, c'est l'ensemble des terrains voisins qui vont progressivement accueillir d'autres implantations industrielles.

Source : Ford.

\section{Les grandes campagnes de la Hesbaye}

Illustration 12 - Othée, village hesbignon, grandes cultures et chemins de remembrement

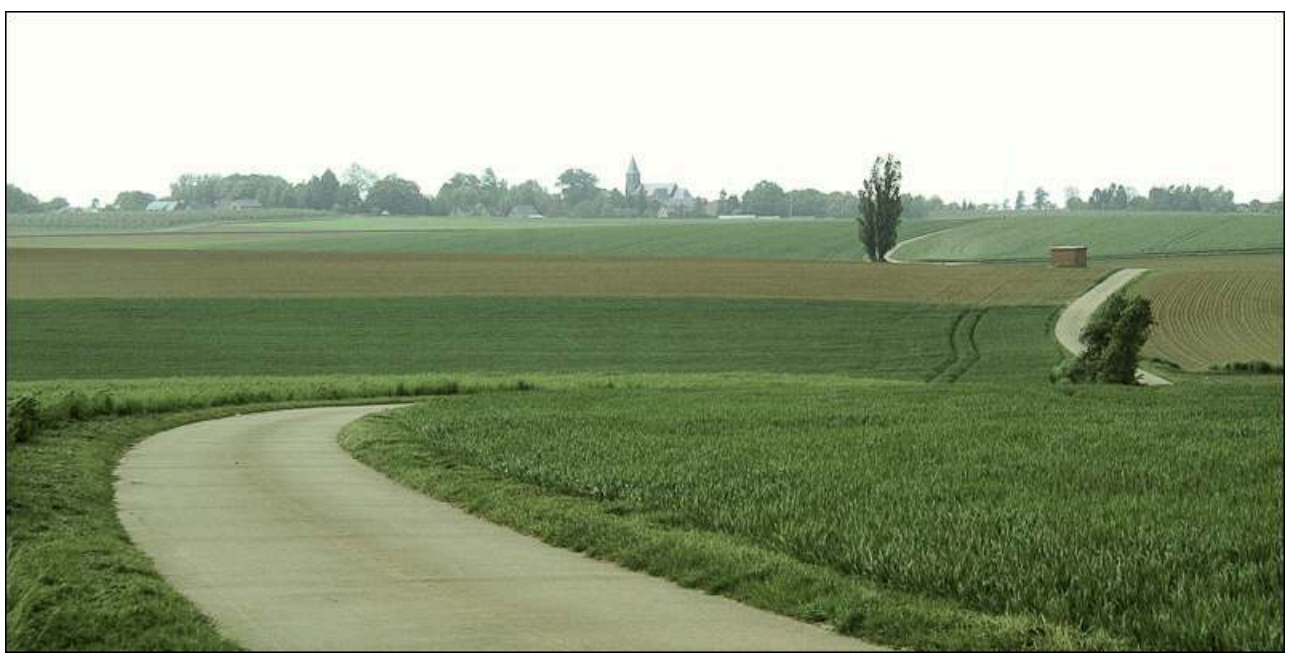

Auteur : C. Neuray, 2008

9 Des parcelles agricoles de grandes dimensions, seulement délimitées par les différences de cultures, reflètent l'excellente valeur du sol limoneux de la Hesbaye, défriché dès le 
Moyen-Âge au profit de la grande propriété foncière nobiliaire. Une photographie du XIXe siècle pourrait nous montrer l'intense activité d'une abondante main-d'œuvre d'ouvriers agricoles ou encore, dans les années 1950, les lourds chariots tirés par des attelages de chevaux brabançons qui sortaient les récoltes des champs, de blé et de betteraves sucrières surtout. Aujourd'hui, seuls quelques agriculteurs juchés sur de puissantes machines travaillent encore dans ces vastes campagnes. Dans les manuels scolaires, les paysages hesbignons sont généralement qualifiés de monotones, essentiellement du fait des grandes cultures permises par le relief, dit calme, d'un plateau, dit mollement ondulé. La Hesbaye, ce sont les vagues des vallons secs, formés sous climat périglaciaire, les cordons arborés qui soulignent le creux de rares cours d'eau, les changements de couleurs des cultures en fonction du rythme des saisons (le vert des blés en croissance au mois de mai sera remplacé par la couleur dorée du blé mur) et partout à l'horizon les silhouettes des villages se laissent seulement deviner par la pointe d'un clocher émergeant d'une couronne de végétation arborée. C'est sans doute la région de la Belgique où la carte topographique actuelle est la plus semblable à celle dressée par Ferraris, pour le compte de l'impératrice Marie-Thérèse d'Autriche, à la fin du XVIII ${ }^{e}$ siècle ${ }^{2}$; mais les cartes topographiques ne permettent évidemment pas de reconnaître le parcellaire, où les remembrements en grands blocs ont remplacé les lanières de l'assolement triennal obligatoire (du moins dans les villages qui n'étaient pas exploités sous l'Ancien régime par de grosses censes seigneuriales indivisibles).

\section{Le Pays de Herve bocager}

Sur les reliefs découpés du moyen plateau du Pays de Herve, dans le nord-est du pays, entre Vesdre et Meuse, l'habitat s'est précocement dispersé, accompagnant dès le XVI ${ }^{\mathrm{e}}$ siècle le développement d'un important bocage herbager, en réponse à celui du textile lainier à domicile. En limite des petites routes locales ou des parcelles de prairies, des alignements de haies, souvent basses, témoignent encore de ce paysage semi-fermé très spécifique, grignoté par la périurbanisation liégeoise. A proximité des habitations, quelques arbres fruitiers subsistent, vestiges des nombreux vergers à hautes tiges sur prairie du XIX ${ }^{e}$ siècle, dont l'image subsiste dans la mémoire collective, accolée à la réputation des produits laitiers et à l'image de « qualité des produits naturels » dont se prévaut l'industrie agro-alimentaire locale - le bon fromage de Herve et le succulent beurre du pays d'Aubel, et en outre le paradis du sirop -, industrie qui assure, plus qu'ailleurs en Wallonie, la valorisation en aval de la production agricole du cru. 
Illustration 13 - Le bocage hervien au dessus de Battice

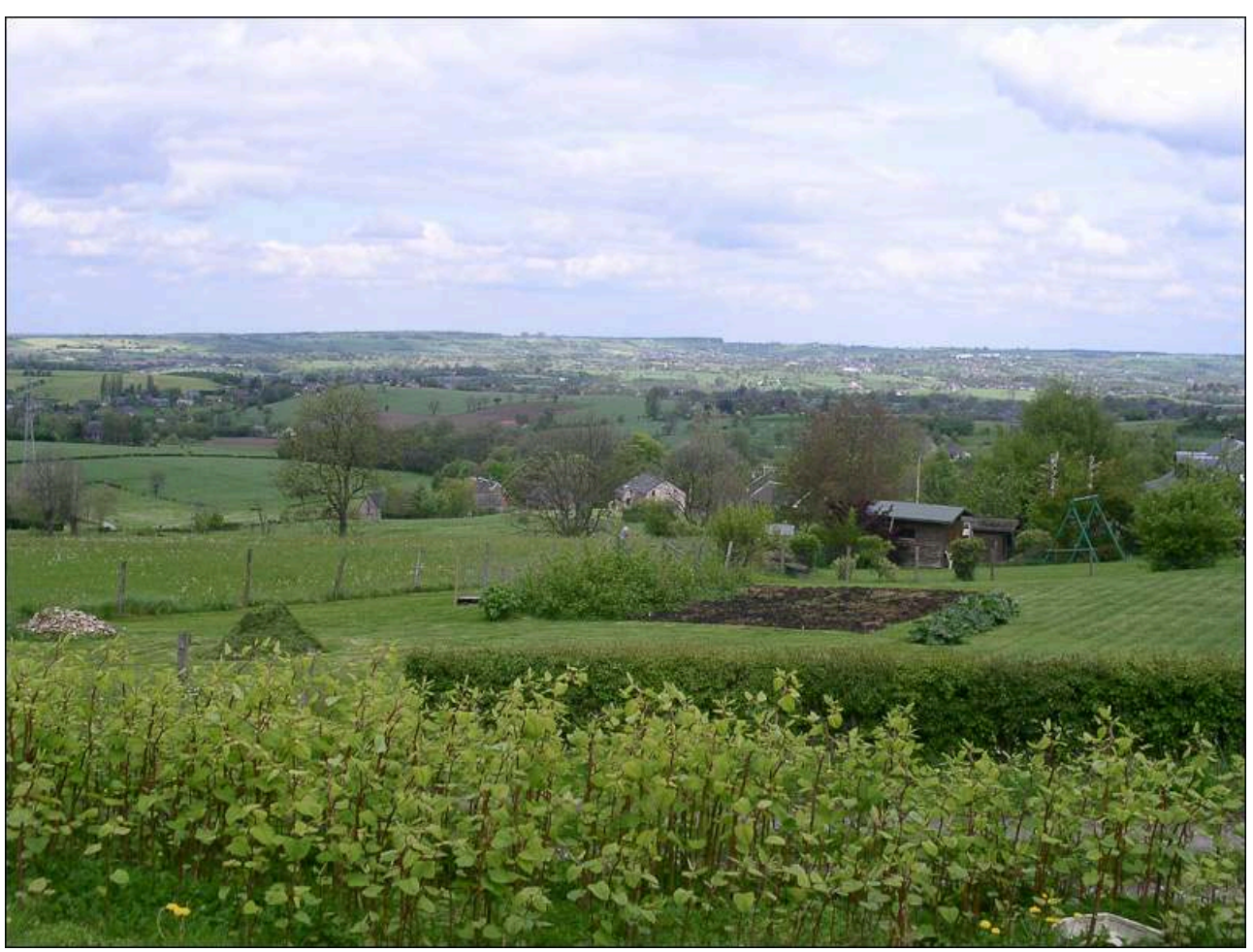

Auteur : C. Vandermotten, 2009

\section{Crêtes et chavées du Condroz}

11 Au sud du sillon mosan, en Condroz, s'allongent, de l'ouest-sud-ouest à l'est-nord-est, les alternances de crêtes psammitiques, les tiges, et de vallées calcaires, parfois sèches, les chavées. Beaux paysages pour livres scolaires, des phénomènes karstiques, un relief appalachien supposé simple à décrire, une articulation affirmée plutôt que statistiquement prouvée entre des villages s'allongeant sous la ligne de crête des tiges, des forêts sur les versants exposés au nord de celles-ci, des prairies dans les fonds calcaires, en tout cas, un pays de grandes propriétés, depuis l'Ancien régime, où les châteaux-fermes sont nombreux. 
Illustration 14 - Dans la chavée, un château-ferme du Condroz, en pierre calcaire : Abée

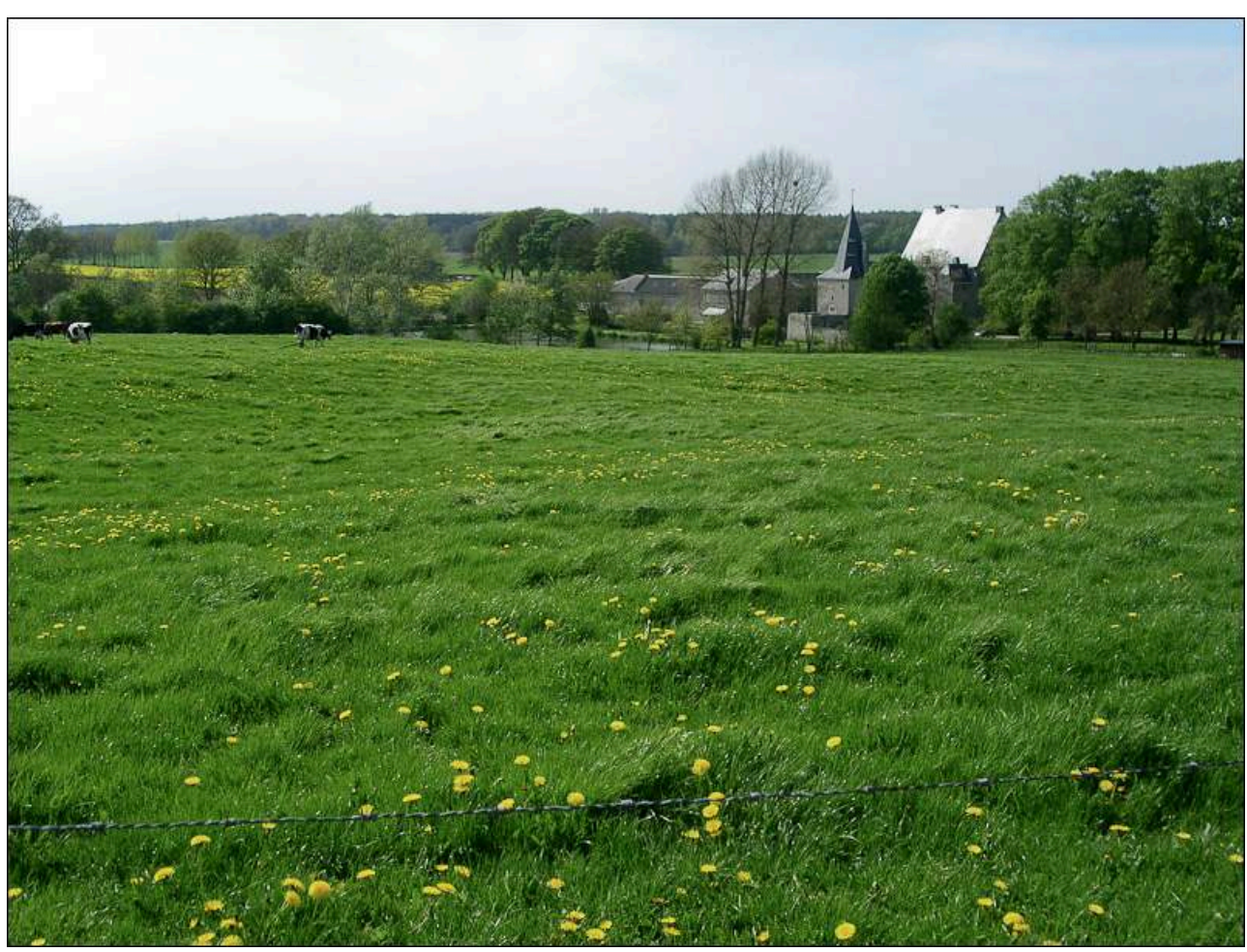

Au fond, le « tige » boisé.

Auteur: C. Vandermotten, 2005

\section{L'Ardenne}

Terres de hauts plateaux, couvertes de forêts ou de prairies, qui furent des terres de culture lorsque l'agriculture était de subsistance, jusqu'au XIXe siècle. La pénéplaine tranche le socle du Dévonien inférieur, voire du Cambro-silurien; elle est entaillée, surtout sur ses bordures, par des vallées encaissées, aux versants densément boisés. C'était la région censée infranchissable par les chars allemands pour les stratèges français de 1939. C'est la montagne et les grands espaces de nature pour les nombreux touristes néerlandais ou flamands, voire le paradis obligé pour des retraités de l'ancienne industrie wallonne. Qui tous aiment se concentrer dans les vallées, près de l'eau, au risque de voir leur caravane entraînée par une prochaine crue. Les villages traditionnels s'implantaient au-dessus de la plaine alluviale, ou sur le plateau, mais un peu à l'abri, sous sa surface sommitale balayée par les vents. 
Illustration 15 - Cens, petit village ardennais du plateau.

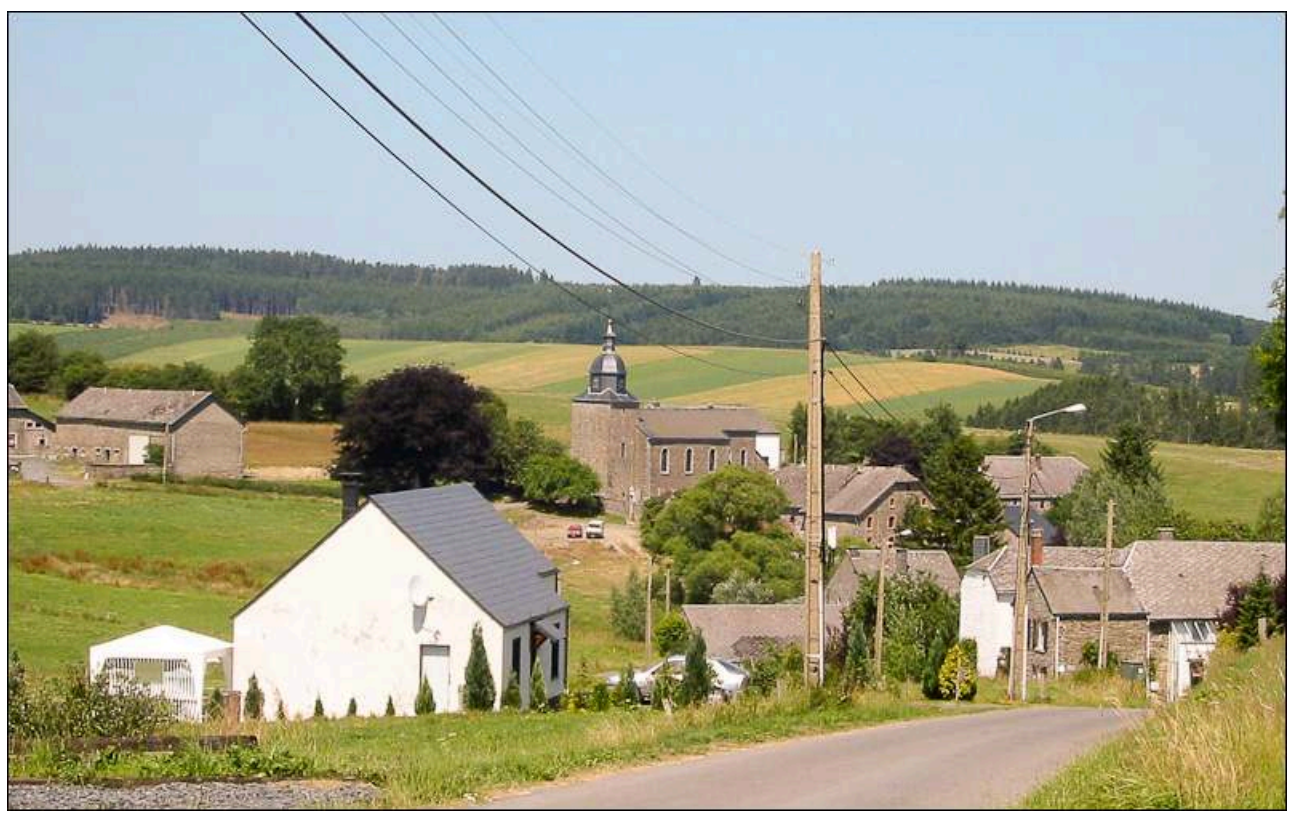

En tas, un peu en dessous des surfaces sommitales, au centre d'une clairière, qui se referme souvent du fait du boisement en épicéas des marges du finage. Maisons grises, quand elles ne sont pas chaulées, construites en psammite ou en schiste ; toits en ardoises, qui ont cédé la place aux plaques d'éternit. Quelques constructions récentes s'étirent le long des routes à la sortie du village. Une ruralité préservée, mais où il reste bien peu d'agriculteurs.

Auteur: C. Neuray, 2003. 


\section{Illustration 16 - Poupehan}

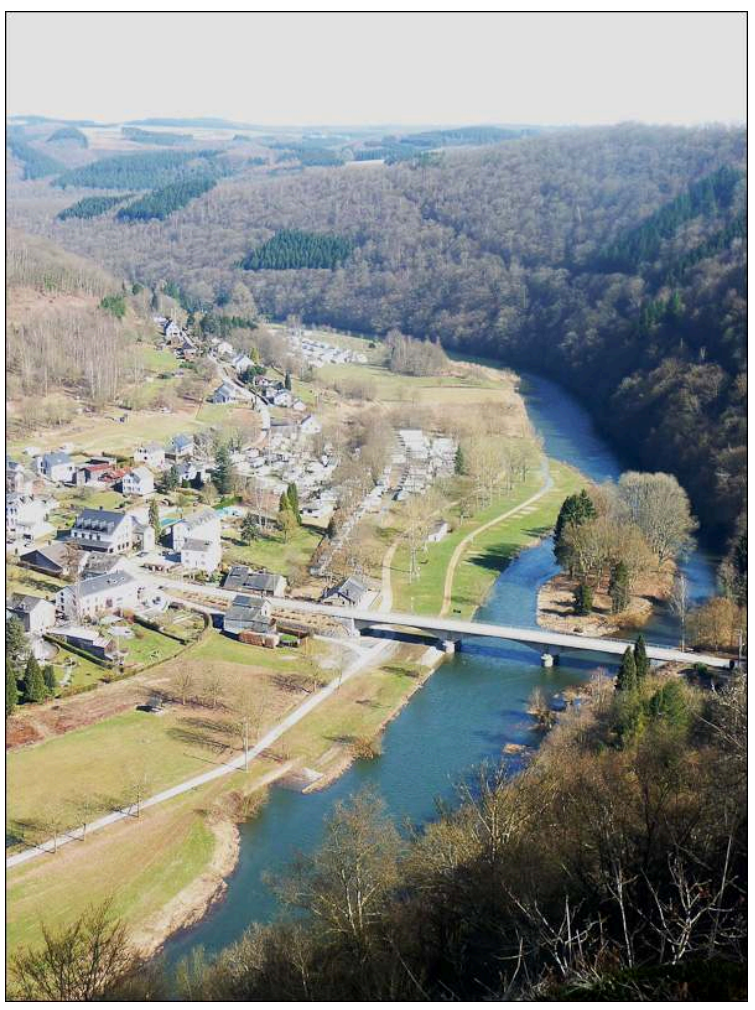

Sur le flanc méridional de l'Ardenne, l'encaissement de la vallée de la Semois à Poupehan. L'ancien village surmontait la plaine alluviale. Les campings ont remplacé les cultures et les séchoirs à tabac. Auteur: C. Neuray, 2010.

Illustration 17 - Le calme des vallées ardennaise : I'Amblève, à Plopsacoo

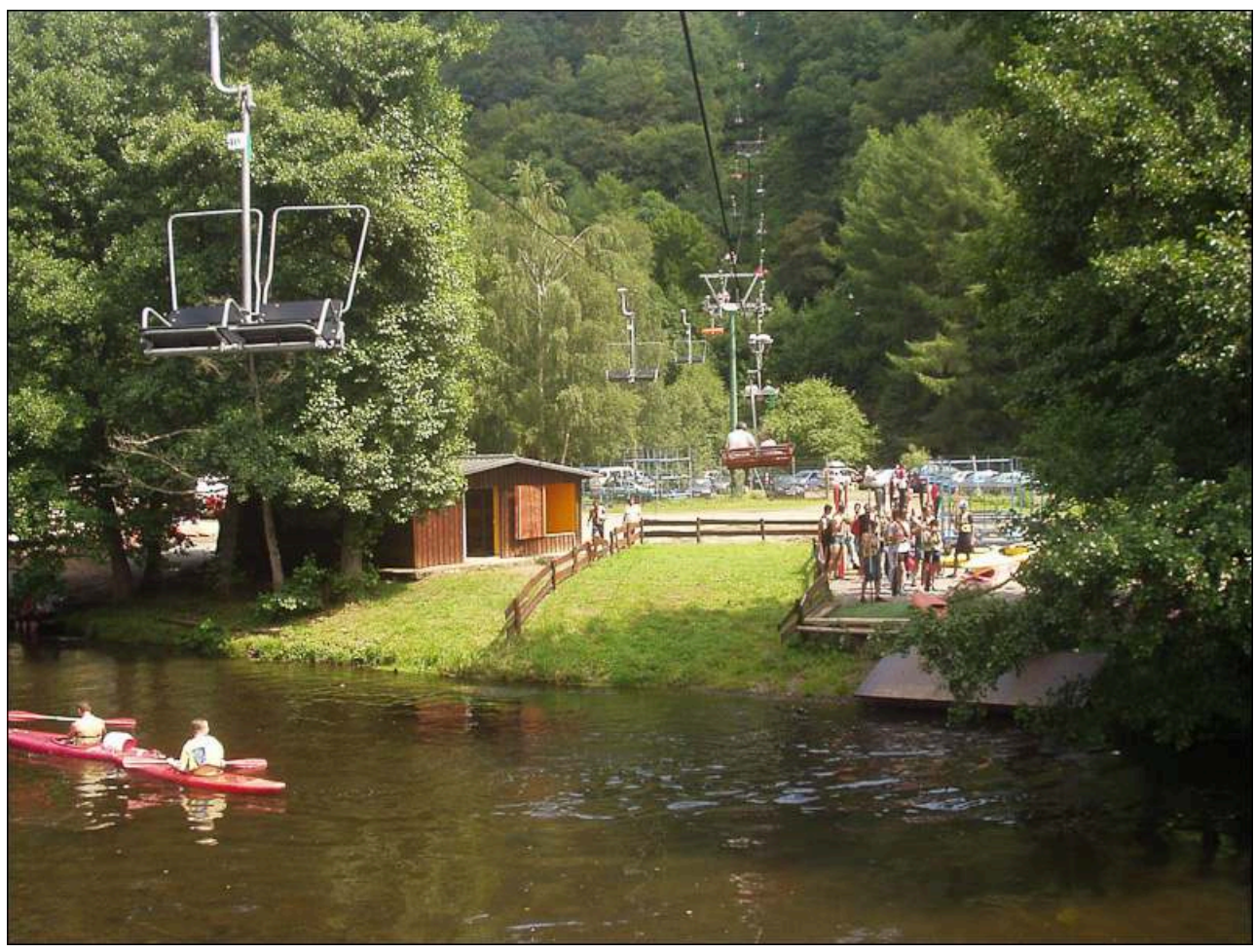

Auteur: Michielvsb, 2004. 


\section{NOTES}

1. La Légende et les aventures héroïques, joyeuses et glorieuses d'Ulenspiegel et de Lamme Goedzak au Pays de Flandres et ailleurs (1867).

2. L'ensemble des feuilles de la carte de Ferraris a récemment été remarquablement réédité en 2009 en un volume : Le grand atlas de Ferraris, Lannoo et Racine, Bibliothèque royale de Belgique.

\section{AUTEURS}

\section{CHRISTIAN VANDERMOTTEN}

Christian Vandermotten, Université libre de Bruxelles, cvdmotte@ulb.ac.be

\section{CLAIRE NEURAY}

Claire Neuray, Université libre de Bruxelles, clneuray@ulb.ac.be 